\title{
Evaluación de proceso de un programa universitario de inglés mediante Blended Learning
}

\section{(Process evaluation of a university English blended learning program)}

\author{
Rodolfo Martinic Lenta \\ École des Hautes Études en Sciences Sociales (Francia) \\ Sergio Urzúa Martínez \\ Ricardo Úbeda Menichetti \\ Roxana Aranda Gengler \\ Universidad de Santiago de Chile (Chile)
}

DOI: http://dx.doi.org/10.5944/ried.22.2.23124

\section{Cómo referenciar este artículo:}

Martinic, R., Urzúa, S., Úbeda, R., y Aranda, R. (2019). Evaluación de proceso de un programa universitario de inglés mediante Blended Learning. RIED. Revista Iberoamericana de Educación a Distancia, 22(2), pp. 305-324. doi: http:// dx.doi.org/10.5944/ried.22.2.23124

\section{Resumen}

En el contexto de globalización actual, las universidades deben enfrentar exigencias de internacionalización que implican proveer a los estudiantes una formación integral orientada al desarrollo de competencias profesionales, entre las cuales el dominio de una segunda lengua ocupa un lugar central. En este marco, durante los últimos años la Universidad de Santiago de Chile, ha implementado un programa de inglés Blended Learning en 48 carreras de ocho facultades, cuyo objetivo consiste en desarrollar competencias lingüísticas y comunicativas a un nivel intermedio. Para esto, el programa cuenta con tres componentes pedagógicos: clases presenciales, tutorías académicas y una plataforma on-line. A fines del año 2017 y comienzos del año 2018, se realizó una evaluación de proceso que buscó conocer, a partir de la perspectiva de los distintos actores involucrados, cómo se ha implementado el programa, poniendo énfasis en el avance hacia el cumplimiento de sus objetivos. La información fue recolectada a través de tres técnicas: una encuesta sobre la experiencia de aprendizaje aplicada a los estudiantes $(\mathrm{N}=780)$, un grupo focal con los docentes del programa y entrevistas semiestructuradas con coordinadores o jefaturas de carrera de la Universidad. Los resultados dan cuenta de una evaluación positiva del programa cuando se observa cada componente en su propio mérito. Sin embargo, al analizar la integración entre estos se constataron cuatro tensiones asociadas 
a: la orientación para el trabajo autónomo, la regulación en la distribución de tutorías, la transición de los contenidos pedagógicos desde la plataforma a la clase y la diferenciación funcional entre los distintos componentes.

Palabras claves: uso didáctico del ordenador; enseñanza superior; enseñanza de lenguas; investigación educativa; autonomía escolar.

\begin{abstract}
In the context of current globalization, universities face demands for internationalization that involve providing students training oriented to the development of professional skills, among which the acquisition of a second language has a central importance. In the last few years, the University of Santiago de Chile has implemented a program of English Blended Learning in arcareers of eight faculties, whose objective is to develop linguistic and communicative skills at an intermediate level. For this purpose, the program consists of three pedagogical components: classrooms lessons, tutoring and an on-line platform. At the end of 2017 and the beginning of 2018, a process evaluation was carried out that sought to know, from the perspective of those immediately involved, how the program had been implemented, emphasizing the progress towards the fulfillment of its objectives. The data collection considered three techniques: a survey about the learning experience of the students $(\mathrm{N}=780)$, a focus group with the teachers of the program and semi-structured interviews with coordinators or heads of the University. The main findings show a positive evaluation of the program when each component is observed by itself. However, the analysis of the integration between these reveals four critical tensions related to: the guidance on autonomous work, the managing of the assignment of tutorials, the transition from the pedagogical contents of the platform to the class and the functional differentiation between the components of the program.
\end{abstract}

Keywords: didactic use of the computer; higher education; tefl; education research; learner autonomy.

La internacionalización de la educación superior se impone como un desafío mayor para las instituciones y exige de estas la generación de nuevos marcos curriculares que permitan a los estudiantes desempeñarse profesionalmente en cualquier lugar del mundo. Para esto, es indispensable la modernización de los planes y programas de estudio, incorporando contenidos tales como: comercio exterior, legislación internacional y normas de calidad internacionales, pero, sobre todo, se hace indispensable incluir el aprendizaje de una segunda lengua (L2), en especial del inglés (Didou y Jaramillo, 2014).

El informe de la OECD del año 2009 sobre educación superior en Chile señala que una de las características prevalente en las instituciones refiere al incipiente desarrollo de las competencias ligadas al uso de un segundo idioma en los programas académicos. En efecto, exceptuando ciertas instituciones, la adquisición 
de competencias intermedias o incluso básicas en inglés no se incluye en los planes de formación. Esta insuficiencia ha sido reconocida por los diversos actores involucrados: entidades de gobierno, empleadores, líderes institucionales, personal académico y estudiantes. El informe también sostiene que, entre los principales obstáculos para hacer frente a este problema, están: la falta de profesores adecuados, la escasa preparación en idiomas durante la educación secundaria y la excesiva carga académica de los programas de las universidades (OCDE, 2009).

En este contexto, la Universidad de Santiago de Chile a partir del año 2014 comenzó a implementar el Programa de inglés Blended Learning (PIBL), el que busca contribuir a la formación integral de los estudiantes por medio del fortalecimiento del inglés. El modelo del programa combina tres dispositivos o componentes pedagógicos: las clases presenciales, una plataforma online y tutorías académicas. Se trata de un programa de inglés general de gran escala, que se aplica en 48 carreras de la Universidad (64\% de la oferta académica universitaria) y cuya finalidad es que los estudiantes alcancen un nivel intermedio en el idioma, equivalente al B1 del Marco Común Europeo de Referencia para las Lenguas (MCE) (Council of Europe, 2018).

El presente artículo exhibe los resultados de una evaluación de proceso sobre el PIBL de la Universidad, que en base a una metodología mixta (encuestas a estudiantes, grupos focales a docentes y entrevistas a actores claves de la institución) buscó conocer el funcionamiento del programa en cuanto a la forma en que se articulan sus componentes y se orienta al cumplimiento de su objetivo.

$\mathrm{El}$ artículo se organiza en seis apartados. En el primero se desarrolla el enfoque de Aprendizaje Combinado, mientras que en el segundo se presentan los elementos teóricos a la base del proceso de aprendizaje de una L2 (Leow, 2015). En el tercero se describe el programa de la Universidad de Santiago y en el cuarto se presenta la metodología utilizada para su evaluación. En el quinto se exponen los principales resultados del estudio y, finalmente, se sugieren mejoras al programa basándose en la evidencia generada.

\section{BLENDED LEARNING EN LA EDUCACIÓN SUPERIOR}

El Blended Learning o Aprendizaje Combinado (AC) emerge como uno de los conceptos pedagógicos más populares a inicios del 2000. A diferencia del Online Learning, que se caracteriza por el uso de internet para acceder a materiales de aprendizaje y posibilitar mediante esta vía la interacción entre profesores y estudiantes (Anderson, 2008), el AC es un enfoque ecléctico que busca obtener los beneficios tanto de la enseñanza presencial tradicional como del aprendizaje online (Güzer y Caner, 2013).

Como la implementación de modelos combinados es cada vez más frecuente y diversa disciplinariamente, se ha generado una importante cantidad de investigaciones, evaluaciones y meta-análisis en torno a la efectividad del AC. En ese sentido, pese a que la evidencia sobre la efectividad del Aprendizaje Combinado 
por sobre los enfoques exclusivamente presenciales o en línea es diversa, ciertas investigaciones dan cuenta de que la aproximación combinada puede ser más efectiva que la tradicional (Ferreira, Vine y Elejalde, 2015; Means, Toyama, Murphy y Baki, 2013).

Entre las ventajas que se suelen atribuir al Aprendizaje Combinado, la mayoría está asociada al rol autónomo y protagónico del estudiante en su proceso de aprendizaje (Gómez, Hernández y Rico, 2009; Tomlinson y Whittaker, 2013) que se relaciona con: a) la posibilidad de trabajar al ritmo y tiempo propio, b) la oportunidad de aprender tanto de materiales específicos del curso como de materiales de otra web, c) la menor dependencia respecto de los docentes, y d) la posibilidad de gestionar el propio conocimiento y transferirlo a posteriores experiencias de aprendizaje de idiomas. En este sentido, si bien se señala que el AC favorece la autonomía de los estudiantes (Serag, 2017), la actitud por el trabajo autónomo se debe potenciar y dirigir, ya que este constituye uno de los elementos centrales para que la metodología sea realmente efectiva.

A su vez, también existirían ventajas de orden práctico, dado que el AC permite alcanzar una mayor cobertura de estudiantes mediante cursos con menos horas presenciales. Aquello evidentemente facilita la enseñanza de una L2 en un contexto en que las instituciones de educación superior a menudo no cuentan con la infraestructura ni los recursos humanos para hacerlo mediante únicamente la modalidad tradicional de aprendizaje en aula (King y Arnold, 2012; Tomlinson y Whittaker 2013).

Si bien la literatura es clara con relación a que no existe la modalidad de AC perfecta, sí se destacan buenas prácticas. En efecto, los cursos de AC deben tener claridad en cuanto a su diseño e identificar el tipo de estudiante, los resultados de aprendizaje, el contenido de lo que se debe aprender, las estrategias de enseñanza y los resultados de las intervenciones educativas (McGee y Reis, 2012). Los autores argumentan que ese conjunto de definiciones debe ser flexible para que el curso sea ajustado y rediseñado considerando el rol que cumple cada componente pedagógico en dicha modalidad. La literatura también señala la necesidad de generar investigaciones institucionales que brinden información relevante y oportuna a los tomadores de decisiones (Graham, Woodfield y Harrison, 2013; Marsh y Johnson, 2013).

Así, la implementación de los cursos se debe concebir como un proceso iterativo, en el que su éxito pasa tanto por la calidad del diseño inicial, como por la capacidad de que estos sean rediseñados con base en evidencia. En consecuencia, la evolución y mejoramiento continuo de los cursos requiere de ciclos de práctica, evaluación y reflexión (Beetham y Sharpe, 2007). El diseño no debe asumir que estudiantes y docentes cuentan con las habilidades técnicas para desarrollar con éxito la modalidad combinada. En este sentido, Porter, Graham, Spring y Welch (2014), recomiendan a las instituciones brindar apoyo técnico necesario a fin de que los participantes aprovechen todo el potencial de esta modalidad. 
Como no existe un diseño efectivo que sea universal, este tiene que ser particularmente sensible a los elementos del contexto (perfil de ingreso de los estudiantes, características de las carreras, cultura institucional, etc.) en el cual será implementado el curso (Sharpe, Benfield, Roberts y Francis, 2006). La decisión en cuanto al enfoque del $\mathrm{AC}$, particularmente en lo que respecta a la relación entre el componente presencial y el online, requiere de una apropiación contextual que considere lo más beneficioso para los estudiantes (Tomlinson y Whittaker, 2013). Las implementaciones tienen éxito, en ese sentido, cuando responden a problemas específicos del contexto educativo (Sharpe et al., 2006).

Un elemento clave en el AC son los docentes, pues el uso de la tecnología en un curso no excluye que los docentes tengan un papel fundamental, lo que conlleva la necesidad de capacitarlos y brindarles los recursos adecuados (Marsh y Johnson, 2013). Los profesores deben alentar la participación de los estudiantes en el entorno virtual, pero a la vez generar modos de interacción colaborativos (Güzer y Craner, 2013). Finardi, Vieira y Schmitt (2016), advierten la necesidad de una mayor presencia de los docentes en el trabajo autónomo de los estudiantes a fin de promover la interacción dentro de la plataforma y retroalimentar oportunamente las actividades de aprendizaje. A su vez, es muy importante la moderación de las expectativas en cuanto a la interacción cara a cara, pues la demanda por presencialidad puede ser una fuente de resistencia y posiblemente, pueda limitar la efectividad del diseño combinado del curso (McGee y Reis, 2012).

En Chile, los estudios han mostrado que la modalidad de AC favorece el aprendizaje de una L2. Los principales hallazgos señalan que se consiguen buenos resultados cuando: a) los estudiantes son entrenados en el uso de herramientas computacionales (García, 2000), b) se promueven estrategias metacognitivas con las que el estudiante puede definir sus propios objetivos y ritmo de aprendizaje (García, Ferreira y Morales, 2011), c) la plataforma ofrece un feedback inmediato de las actividades realizadas que sirven al estudiante para conocer sus errores y monitorear sus avances (Morales y Ferreira, 2008) y, d) se incluyen una serie de estrategias que permiten a los estudiantes interactuar entre sí, con el docente o con la plataforma (Bañados, 2006).

\section{EL PROCESO DE APRENDIZAJE DE UNA L2}

El diseño curricular de un programa que adopte el AC debe estar respaldado por un sustento teórico que dé cuenta de los procesos inherentes a la adquisición de una L2. En este sentido, el proceso de transformación de input lingüístico en output lingüístico, parece ser un asunto crucial para el aprendizaje de una L2.

El proceso de adquisición de una L2 contempla los siguientes elementos (Gass, 1997; Gass y Selinker, 2008): 
- Información lingüística (input). Información sobre la L2 a la que es expuesto un aprendiz, contiene los elementos lingüísticos y semánticos a los cuales debe poner atención el aprendiz en el proceso de adquisición de una L2.

- Información internalizada (intake). Refiere al input que puede ser incorporado por un aprendiz para quedar potencialmente disponible para el procesamiento en el sistema de representación interno.

- Sistema de representación interno (internal system). Considera el momento en que los elementos aprendidos total o parcialmente son almacenados.

- Producción lingüística (output). Constituye la evidencia del estado de representación interna que existe en el sistema de interlengua.

Leow (2015) describe las etapas del proceso de adquisición de una L2, incorporando las nociones de "proceso" y "producto":

- El procesamiento de información lingüística (Input Processing). Constituye la etapa inicial del proceso de adquisición de una L2 e implica: (1) un grado de competencia por los recursos de atención que deben ser activados con relación a la información recibida; (2) una adecuada estimación de la carga cognitiva y del esfuerzo mental requeridos para procesar la información recibida, y (3) reconocer que los recursos de atención a la información recibida es parte de una arquitectura de recursos cognitivos.

- Procesamiento de información internalizada. VanPatten (2004) define el concepto de información internalizada (intake) como la parte de la información lingüística (input) que ha sido procesada en la memoria de corto plazo y que está disponible para el procesamiento total y la potencial incorporación al sistema interno de interlengua. Siguiendo a Leow (2015), es posible afirmar que: (1) solo una parte de la información lingüística (input) puede convertirse en información internalizada (intake) debido a las limitaciones respecto de los recursos de atención que puede experimentar un aprendiz de una L2, (2) no toda la información internalizada (intake) es procesada en etapas siguientes, y (3) la parte procesada puede potencialmente ser incorporada al sistema interno de representación lingüística (internal system). En esta etapa operan variables que incluyen el procesamiento dirigido de datos y procesamiento conceptual (Robinson, 1995), las conexiones entre forma y significado (VanPatten, 2004), y formación, modificación, rechazo y comprobación de hipótesis (Gass, 1997).

- Procesamiento de conocimiento a representaciones internas. Constituye la etapa final en la adquisición de una L2 y comprende la manipulación de las representaciones lingüísticas internas del aprendiz, junto con representaciones adicionales que determinan las características fonológicas, sintácticas, semánticas, culturales, pragmáticas y discursivas que se activan a través de la producción lingüística de una L2. 
Así, el aprendizaje de una L2 constituye un proceso en el que se producen diversos cambios en la cognición: los aprendices intentan crear una nueva representación para la gramática de la L2, internalizar esa información y reestructurarla si es necesario, mientras desarrollan su capacidad de comprender y producir en tiempo real la L2 (Leow, 2015).

\section{PROGRAMA DE INGLÉS BLENDED LEARNING (PIBL) ${ }^{1}$}

El Programa de Inglés Blended Learning (PIBL) de la Universidad de Santiago de Chile, es una iniciativa institucional que busca garantizar la formación integral de sus estudiantes a través del fortalecimiento del inglés. Su objetivo consiste en el desarrollo de competencias lingüísticas y comunicativas en inglés a nivel intermedio ("B1" o "ALTE 2")2, mediante cuatro niveles semestrales de carácter semi-presencial (A1, A2, B1-, B1) (Consejo Europeo, 2018). Cada nivel considera 25,5 horas de trabajo presencial en aula y 64,5 horas no presenciales de trabajo autónomo en plataforma.

El programa se estructura a partir de tres componentes pedagógicos: clases, plataforma y tutorías.

- Clases presenciales. Componente principal del programa, se realizan sesiones semanales obligatorias de dos horas pedagógicas y tienen el objetivo de desarrollar las competencias comunicativas de cada nivel. Las clases tienen un énfasis en la comunicación oral, para lo cual las clases propenden a "activar" los contenidos que los estudiantes previamente revisaron en la Plataforma.

- Plataforma English Discoveries Online (EDO)3. Dispositivo E-learning, se concibe como el "libro de clases" del programa, en el cual los estudiantes llevan a cabo el trabajo autónomo requerido en cada nivel. Para esto contempla -entre otros recursos- el uso de videos multimedia con expresiones idiomáticas y la participación en comunidades interactivas multimodales.

- Tutorías. Componente complementario y de carácter voluntario que busca brindar una atención personalizada a los estudiantes para reforzar y consolidar las competencias lingüísticas y comunicativas. Su diseño permite atender de forma presencial hasta 20 estudiantes por turno. Las tutorías se orientan a reforzar y consolidar las competencias lingüísticas y comunicativas revisadas en las clases presenciales.

La estructuración de los componentes del PIBL considera criterios en cuanto a las acciones propias de una modalidad combinada (Marsh y Johnson, 2013): a) planificación de trabajo independiente sobre una estimación de carga de trabajo semanal predeterminada, b) criterios de flexibilidad para la planificación de los tiempos de trabajo autónomo por parte del estudiante, c) generación de instancias de trabajo colaborativo y d) instancias de provisión de retroalimentación correctiva y generación de instancias de autocorrección. Como el componente de las clases 
presenciales es el eje articulador, los aspectos c) y d) son guiados por el docente del curso.

Siguiendo el modelo de Loew (2015), en el PIBL la plataforma cumple el rol del entorno de aprendizaje donde los estudiantes se exponen a través de un trabajo autónomo a la información lingüística (input). Las clases, por su parte, buscan generar las condiciones para facilitar el proceso de internalización del input a través de la activación en la comunicación oral, para así permitir que se fije en la memoria de corto plazo y quede disponible para el procesamiento total y la potencial incorporación a la interlengua. Por último, las tutorías se conciben como instancias adicionales para reforzar el trabajo en clases y facilitar el procesamiento desde el conocimiento a las representaciones internas, junto con las representaciones adicionales que permitirían trabajar las características fonológicas, sintácticas, semánticas, culturales, pragmáticas y discursivas activadas a través de la producción lingüística de una L2.

Actualmente, el programa se implementa en 48 carreras (64\% de la oferta académica universitaria), dependientes de ocho facultades o unidades académicas: Bachillerato, Administración y Economía, Ciencias Médicas, Derecho, Humanidades, Ingeniería, Química y Biología y Tecnológica.

\section{MÉTODO}

\section{Diseño de la evaluación}

El estudio se planteó como una evaluación de proceso con enfoque formativo, vale decir, estuvo internamente orientada para proporcionar información sobre los aspectos específicos en que el programa puede mejorar. En ese sentido, en lugar de buscar resultados estandarizados o de imponer criterios de logro externos, este tipo de evaluación recopila información útil para responder a las preguntas más relevantes del programa y, de esta forma, ayudar a las partes interesadas y a los responsables de la toma de decisiones, a resolver los desafíos inmediatos (Norris, 2016).

La orientación de la evaluación se fundamenta en las inquietudes de los responsables del programa ${ }^{4}$, respecto a la contribución específica de cada componente al aprendizaje de una L2 y la interrelación que se genera entre estos en la implementación. En este marco, la evaluación de proceso desarrollada tuvo por objetivo conocer el funcionamiento de cada componente pedagógico y el modo en que se articulan en la implementación del programa.

A partir de ese objetivo, se formularon las siguientes preguntas directrices:

- ¿Cuáles son los usos que le otorgan los estudiantes a los componentes pedagógicos del programa?

- ¿Cómo valoran el aporte de cada componente a su proceso de aprendizaje de la L2? 
- ¿Cuáles son las tensiones que observan estudiantes, profesores y actores claves en la articulación de los componentes pedagógicos?

\section{Instrumentos}

Para responder a las preguntas directrices de la evaluación se emplearon tres instrumentos:

a. Encuesta Experiencia de Aprendizaje aplicada a estudiantes. Orientada a conocer el uso que les dan los estudiantes a los componentes para el desarrollo de las competencias lingüísticas en la L2, así como también la percepción respecto de la articulación que existe entre estos. Su población objetivo fueron los estudiantes que al segundo semestre del 2017 cursaban el último nivel del PIBL. Tuvo un carácter censal y fue respondida por 780 estudiantes, alcanzando una tasa de respuesta del $78 \%$. La encuesta fue construida ad-hoc para la evaluación y se validó mediante juicio de expertos (profesionales del Área de Evaluación de la Vicerrectoría Académica y del PIBL). Esta fue aplicada durante la segunda semana de noviembre de 2017 en horario de clases.

b. Grupo focal con los docentes del programa. Dirigido a conocer la experiencia de los docentes respecto de los componentes y de la articulación entre estos. Se realizó el día 14 de diciembre de 2017 y contó con la participación de 8 docentes, que corresponden a un tercio de los docentes que participan del programa. Se aseguró el anonimato y el tratamiento confidencial de la información brindada. La conversación duró una hora (aproximadamente), fue grabada íntegramente y luego transcrita para su análisis.

c. Entrevistas semi-estructuradas a informantes claves (jefes de carreras y coordinadores de inglés). Su objetivo fue conocer la experiencia de las carreras y facultades con el programa. La duración de las entrevistas en promedio fue de aproximadamente 30 minutos y fluctuó considerablemente en función del grado de conocimiento e involucramiento de los participantes con el PIBL. Cada entrevista fue posteriormente transcrita para el análisis.

\section{Procedimiento y análisis de Información}

El estudio tiene un carácter mixto: cuantitativo, ya que se realizó un análisis descriptivo (números absolutos y porcentajes) de cada una de las preguntas de la encuesta "Experiencia de Aprendizaje"; y cualitativo, por el análisis realizado sobre el grupo focal y las entrevistas semiestructuradas. Estas fueron analizadas de acuerdo con los procedimientos de codificación de la grounded theory (Strauss y Corbin, 2002), mediante los cuales se fragmentó la información recogida en múltiples categorías, que luego fueron integradas de manera gradual en conceptos de mayor abstracción y capacidad comprensiva. 
Con el objetivo de reducir sesgos y mejorar la comprensión del fenómeno, el proceso de análisis implicó la triangulación de las fuentes de información (Benavides y Gómez-Restrepo, 2005). De esta forma, se buscó conocer el funcionamiento del programa considerando la perspectiva de estudiantes, docentes, coordinadores y directivos de carrera.

\section{RESULTADOS}

Los resultados se presentan en dos apartados: el primero, da cuenta de la valoración y usos de cada componente, mientras que el segundo, presenta las tensiones en la implementación del PIBL, específicamente aquellas asociadas a la articulación entre sus componentes.

\section{Valoración y usos declarados de los componentes}

A nivel general, los participantes del estudio tienen una percepción positiva al evaluar cada componente en su propio mérito. En el gráfico 1 se presentan los usos que los estudiantes hacen de cada componente. Se aprecia que a las clases presenciales se les reconoce una mayor importancia en el aprendizaje del idioma, destacando su aporte a la producción oral de la L2.

Gráfico 1. Usos asociados a cada componente PIBL

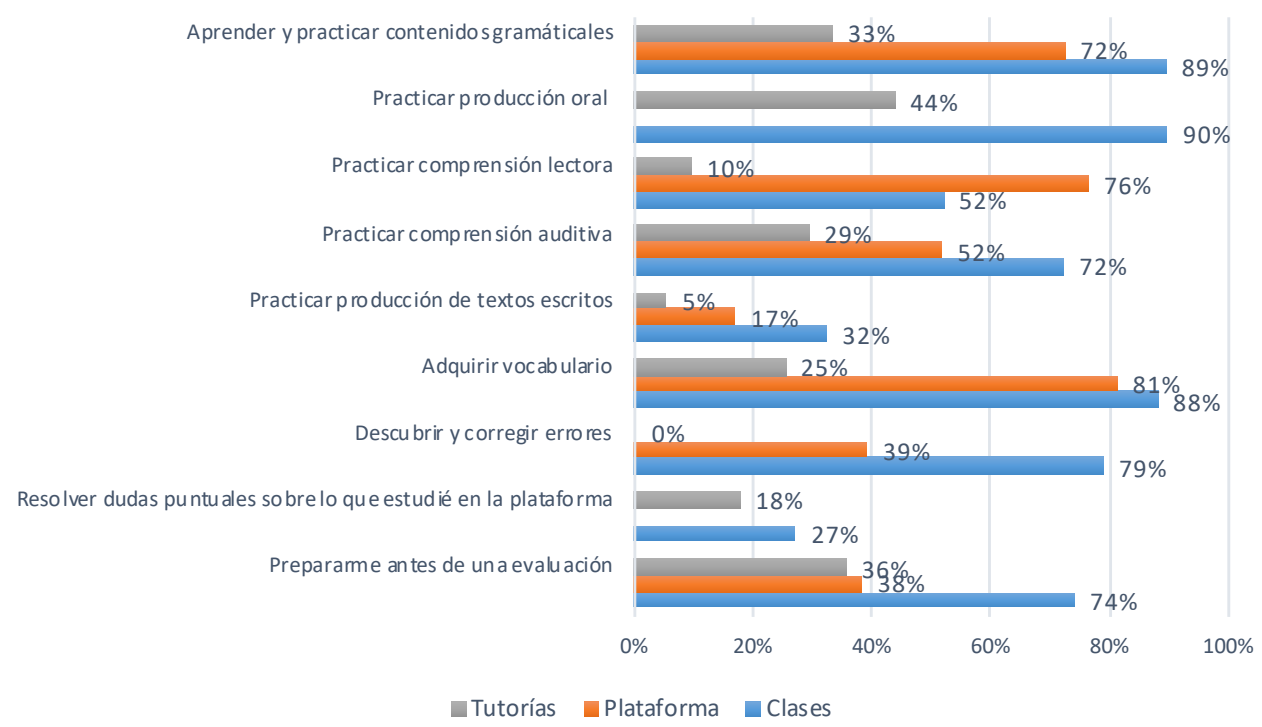

Fuente: Elaboración propia en base a encuesta. 
Esto es consistente con el testimonio de los docentes, quienes consideran que el énfasis en la expresión oral constituye una de las principales fortalezas del programa.

El hecho de que en clases tratemos de hacerlo lo más comunicativamente posible, yo encuentro que eso también es una fortaleza. Los alumnos agradecen tener esa instancia de poder hablar inglés, a diferencia de otros profes $u$ otras instancias donde todo es pizarra, anoten la fórmula, así se hace, ejercicio (Docente, grupo focal)

La plataforma, en tanto, destaca por aspectos relacionados con el trabajo individual de los estudiantes (adquisición de vocabulario, práctica de la comprensión lectora y aprendizaje y ejercitación de contenidos gramaticales). Sin embargo, el cuarto uso más declarado en la encuesta entrega luces sobre una cierta "instrumentalización" del componente, ya que un $67 \%$ de los estudiantes señala que la utilizó para la realización de los controles del curso. Aquello fue congruente con el relato de los docentes, quienes afirman que los estudiantes rinden esos controles muchas veces solo para terminar el curso y obtener una calificación de aprobación.

Finalmente, las tutorías son una instancia para ejercitar la comunicación oral, aunque también tiene un uso estratégico vinculado al estudio previo a exámenes. En efecto, entre los principales usos reportados están: la práctica de la producción oral, la preparación antes de una evaluación y el reforzamiento de las habilidades menos desarrolladas. Sin embargo, y de acuerdo a lo señalado en la encuesta, una de las limitaciones de las tutorías es que si bien tienen un alto nivel de conocimiento por parte de los estudiantes (96\%), sólo un poco más de la mitad de los estudiantes afirma haber asistido alguna vez durante los cuatro niveles del programa (54\%). Esto es así ya que las tutorías funcionan bajo una lógica de "libre demanda", permitiendo que los estudiantes puedan seguir profundizando en el aprendizaje del idioma de manera personalizada, en función de las habilidades y/o contenidos que ellos definan.

Las tutorías se conciben principalmente como una instancia de nivelación, la que es utilizada por los estudiantes con mayores dificultades para aprender el idioma, mejorando así su desempeño académico en los cursos del PIBL. Pero también, según los docentes, las tutorías operan como una instancia de perfeccionamiento para quienes cuentan con la motivación de seguir progresando fuera del espacio de las clases presenciales.

Hay gente que tiene un muy buen nivel, pero que quieren ampliarse más en un área, entonces ellos también aprovechan, en menor medida sí que los que están conscientes de que tienen problemas. Pero también ocurren esos casos de que quieren seguir mejorando, porque al final es como si fuera una clase particular (Docente, grupo focal) 


\section{Articulación de los componentes pedagógicos del programa}

A continuación, se presentan cuatro aspectos críticos de la articulación entre los componentes del PIBL identificados en la evaluación:

\section{El aprendizaje autónomo}

La flexibilidad del PIBL y la correlativa autonomía en el aprendizaje es un elemento que el programa intenciona y que las carreras valoran de forma positiva. $\mathrm{Al}$ entregar la posibilidad a los estudiantes de gestionar su tiempo de estudio y cumplir de forma remota con parte de las exigencias del curso, estos pueden dar mayor prioridad a las asignaturas propias del plan de estudio de su carrera. Sin embargo, esta autonomía tiene un carácter altamente ambivalente, pues también es reconocida como una de las principales dificultades en la implementación del programa.

En efecto, pese a que el programa considera una carga de trabajo autónomo equivalente a cuatro horas semanales, este no considera orientaciones sobre cómo los estudiantes deben llevarlo a cabo. Tampoco se realiza un seguimiento para garantizar su cumplimiento efectivo, ni se han establecido lineamientos para que los estudiantes puedan aprovechar al máximo este dispositivo pedagógico. El programa, de esta forma, asume la premisa del trabajo autónomo del estudiante con la expectativa de que cumpla por sí mismo.

En esta misma línea, los docentes señalan que la plataforma no es utilizada por los estudiantes de la manera en que se pensó en su diseño inicial, a saber, como "libro de clases”, el que se revisa previo a cada sesión. La consecuencia de aquello para los docentes consultados es que han debido comenzar a trabajar con los estudiantes los contenidos de la plataforma en el espacio de la clase presencial.

Inicialmente se consideraba que la plataforma era el principal input de contenido. La parte de presentación de la clase debía ir en la plataforma. El alumno debía hacer la unidad antes de la clase e ir a clases a practicar, a consolidar, automatizar [...] y eso no pasa [...], por eso decidimos tomar elementos de la plataforma e incluirlos en la clase (Docente, grupo focal)

De este modo, pese a que la plataforma en sí misma es bien evaluada tanto en su diseño como en términos técnicos y/o pedagógicos, esta presenta dificultades en relación a los mecanismos con los que se cuenta para asegurar que el trabajo que los estudiantes realizan en ella se lleve a cabo tal y como fue ideado.

\section{La regulación en las tutorías}

Las tutorías son entendidas por los docentes como un espacio al cual los estudiantes pueden recurrir (voluntaria e ilimitadamente) para trabajar el contenido 
que deseen. Cada profesor tiene su propia forma de citar a los estudiantes: algunos esperan que estos por iniciativa propia soliciten o se inscriban en las tutorías, mientras que otros citan directamente a quienes tienen mayores dificultades con el idioma.

Es que ahí también depende del profe. Yo por ejemplo no espero que ellos me pidan tutorías, yo identifico a los que tienen problemas y los cito a tutorías. Les digo 'tú tienes dificultades, tienes que estudiar o si no te vas a echar el ramo'. Entonces, tampoco es esperar que vengan a pedir y en ese sentido funciona (Docente, grupo focal).

Las tutorías, de esta manera, no constituyen una instancia de aprendizaje regulada bajo criterios comunes. Las formas en que los estudiantes acceden (citación/solicitud) depende de los docentes, mientras que los contenidos tratados se definirán en función de las debilidades observadas por el docente (citación) o del requerimiento específico del estudiante (solicitud).

Además, se ha observado que la asistencia a las tutorías se concentra en los estudiantes de una sola facultad de la Universidad, precisamente una de las que posee mayores exigencias de ingreso a sus estudiantes. Esto invita a pensar el papel que deben cumplir las tutorías en el programa, pues la evidencia señala que cuando los servicios de apoyo académico son voluntarios y desregulados pueden quedar excluidos aquellos que por diversos factores carecen de motivación o son académicamente más “débiles" (Warren, 2002).

El que utiliza ese recurso, bien porque de verdad va a llegar, a lo mejor no va a llegar a los compañeros, pero va a llegar al nivel mínimo para pasar la prueba. En cambio, el que le cuesta se queda callado en clases, no participa y más encima no va a clases... Ah no, ese ya se tiró esos son siempre mis reprobados (Docente, grupo focal).

Así, aunque las tutorías pueden ser utilizadas por algunos estudiantes para nivelar sus habilidades y conocimientos en inglés, en la medida en que no se enfocan deliberadamente en los estudiantes que tienen mayores dificultades, puede que no se les esté brindando una oportunidad para que se involucren y adopten un grado mayor de compromiso en relación con el aprendizaje del idioma.

\section{Transición plataforma-clase de los contenidos}

Los docentes reconocieron algunos problemas con relación al tránsito de los contenidos gramaticales de la plataforma a las clases presenciales. Esta tensión se observa particularmente en aquellas unidades que acentúan los contenidos gramaticales, ya que los docentes tienen dificultades para conciliarlas con el énfasis 
en la comunicación oral de las clases presenciales. Parte de los docentes advierten que existen contenidos en la plataforma, cuya utilización es sobre todo escrita y que no aportan a una comunicación fluida del idioma.

Es difícil decirles, passive voice, voz pasiva me dicen 'profe, ¿esto cuándo lo voy a usar?' En la práctica no se usa mucho oralmente, pero sí cuando escriban reportes o cosas así. [...], hay contenidos en la plataforma que de repente no son vitales para que los alumnos tengan una fluidez comunicativa. Son demasiado gramaticales y cuesta en clases implementar actividades para que ellos la usen comunicativamente (Docente, grupo focal).

Para los estudiantes ese tipo de contenidos carece de sentido, debido a que no se visibiliza su utilidad en la comunicación cotidiana. Por su parte, los docentes si bien valoran el énfasis en la comunicación oral que prevalece en el PIBL, consideran necesario fortalecer el trabajo gramatical, ya que este se encuentra a la base de las otras habilidades.

\section{Diferenciación funcional entre los componentes}

El estudio dio cuenta de que existiría una escasa diferenciación funcional de los componentes del programa. En el gráfico 2, se presentan las respuestas de los estudiantes respecto de la contribución de cada componente al logro de las habilidades en un dominio de inglés $\mathrm{B} 1^{5}$.

Gráfico 2. Contribución de los componentes al desarrollo de las competencias lingüísticas

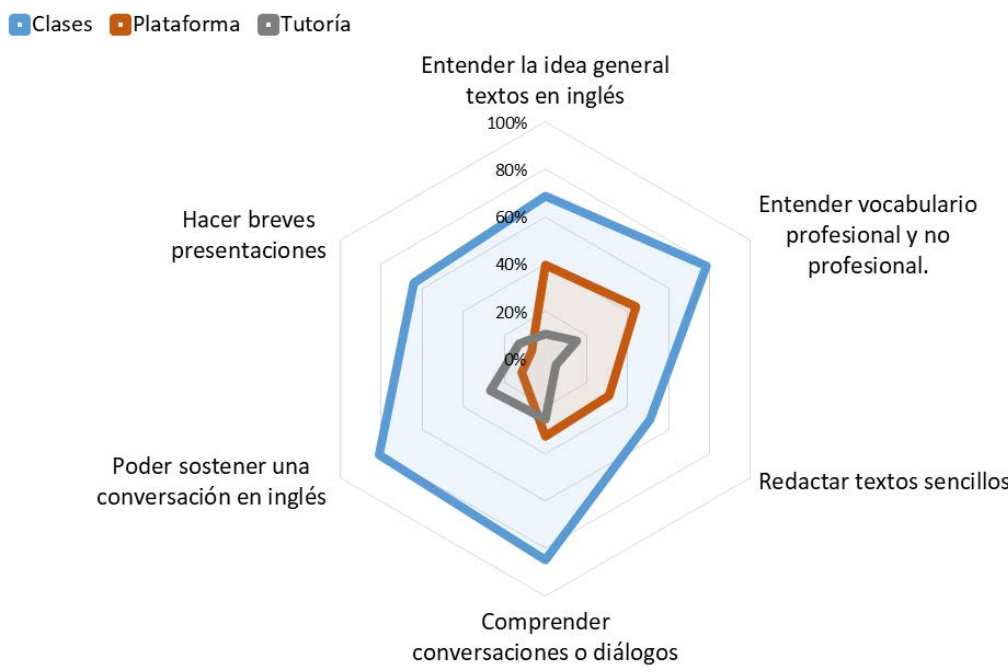

Fuente: Elaboración propia en base a encuesta 
En general, se observa que las clases presenciales hacen la mayor contribución al desarrollo de todas las habilidades. Su mayor aporte se aprecia en la comprensión de conversaciones o diálogos (85\%), en la capacidad de sostener conversaciones cotidianas $(81 \%)$ y en la comprensión de vocabulario profesional y no profesional (78\%). La plataforma, en tanto, contribuye con mayor fuerza al entendimiento de la idea general de los textos (44\%) y a la comprensión de vocabulario (40\%), mientras que las tutorías contribuyen a la capacidad de sostener una conversación (27\%) y a la comprensión oral (26\%). No obstante, el aporte de las tutorías a esta última habilidad es menor que el que realizan los otros dos componentes.

El gráfico de zonas permite observar que las clases presenciales son consideradas como un aporte a todas las competencias lingüísticas y a la vez es el componente que más contribuye a cada una de estas. La principal consecuencia de esto es que las otras vías de aprendizajes diseñadas en el programa estarían siendo subutilizadas.

Dado esto, se puede sostener que existe una falta de diferenciación funcional de los componentes pedagógicos, ya que lo que se debiese esperar de una modalidad AC es que exista una tributación diferenciada de los diversos componentes a cada una de las competencias lingüísticas involucradas.

\section{CONCLUSIONES}

Este trabajo presentó los resultados de una evaluación de proceso del PIBL, que mediante una metodología mixta (encuestas, entrevistas y grupo focal), se orientó a conocer su funcionamiento y la articulación entre sus componentes. En este contexto, los principales hallazgos de la evaluación indican que cuando se evalúa cada componente en su propio mérito, la percepción de estudiantes, docentes y coordinadores es positiva; no así cuando la evaluación se orienta a la articulación entre las clases, las tutorías y la plataforma. En este caso, se observaron cuatro tensiones en la implementación del programa: la primera, asociada al aprendizaje autónomo; la segunda, a la regulación de las tutorías; la tercera, a la transición entre la plataforma y la clase presencial y la última, a la diferenciación funcional de los componentes.

El estudio permite extraer conclusiones en la siguiente dirección. En primer lugar, la valoración de cada componente pedagógico contrasta con las dificultades que presenta la articulación entre estos. Aquello permite inferir que los esfuerzos en torno al diseño han estado más centrados en elementos internos de cada componente que en el modo en que estos se interrelacionan, privilegiando el buen funcionamiento técnico por sobre las normas que regulan la articulación. Pareciera que, en ese sentido, el todo es más que la suma de las partes.

En estrecha relación, en segundo lugar, se observa que los diferentes componentes tienen un bajo nivel de diferenciación en cuanto a sus funciones. En efecto, las clases se evidencian como el componente que permite el desarrollo de casi todas las habilidades, principalmente porque, junto con sus funciones, realiza 
las atribuidas a la plataforma. Las tutorías, por su parte, del mismo modo que las clases, se enfocan en la práctica de la comunicación oral, pero como se trata de una instancia voluntaria con cupos limitados en la cual solo una parte de los estudiantes participan, la contribución a los otros componentes es poco clara.

Por último, la plataforma presenta dificultades para fungir el rol del "libro de clases" del programa. Lo anterior, por un lado, porque el trabajo autónomo que los estudiantes realizan parece ser escaso y, por otro, por el difícil tránsito de contenidos que requieren mayor profundización en su gramática o que no poseen un uso predominante en la oralidad. Como consecuencia, la activación de los contenidos de la plataforma en las clases parece no ocurrir de acuerdo a lo planificado.

A partir del modelo de Loew (2015), podría afirmarse que existen dificultades en el programa en relación al rol de la plataforma como entorno de aprendizaje en el cual los estudiantes acceden a la información lingüística (input) de manera regular y constante. Como se observó, el procesamiento del input depende de grados óptimos de atención selectiva por parte de los aprendices y de los rasgos lingüísticos presentes en la información lingüística recibida (Gass y Selinker, 2008), lo que sería solo parcialmente cumplido. Como consecuencia de lo anterior, se generarían problemas para garantizar en las clases las condiciones del proceso de internalización del input e incorporarlo en la memoria de corto plazo y potencialmente en la interlengua. Esto releva el lugar de importancia que tiene en la modalidad combinada el trabajo autónomo de los estudiantes, pero también de los lineamientos de cómo llevarlo a cabo y del acompañamiento que se le realiza por parte de profesores.

De acuerdo a las tendencias observadas en la literatura sobre AC y la implementación de esta modalidad, no existirían recetas que garanticen su efectividad. No obstante, coinciden en afirmar que los diseños deben concebirse de manera "iterativa", siendo constantemente revisados para realizar modificaciones y ajustes al plan original. Esto debe llevarse a cabo considerando los desafíos específicos que surgen del contexto educativo.

En este marco la presente evaluación nutre de información a los tomadores de decisiones para que incorporen mejoras, optimizando el funcionamiento y alineando los aportes de cada componente.

Con base en la evidencia, a nivel general se sugiere definir el diseño de cada uno de los componentes pedagógicos del programa, diferenciando sus funciones y estableciendo el modo en que se relacionan entre sí para el logro de los aprendizajes. También se recomienda generar mecanismos de monitoreo que permitan velar por la articulación efectiva entre los componentes, que entreguen alertas oportunas para reajustar o mejorar la implementación. Un aspecto particularmente sensible a este respecto es el trabajo autónomo de los estudiantes en el componente online, el cual debe ser supervisado permanentemente, ya que de este depende en gran medida el buen funcionamiento del programa. Por último, se sugiere que las tutorías sean focalizadas en los estudiantes que presenten más dificultades con la L2. 


\section{NOTAS}

1. Información del programa y de los cursos que lo componen, se puede encontrar en: http://blearning.usach.cl/bienvenido-blended-learning

2. Según el MCE y la The Association of Language Testers in Europe, respectivamente.

3. Más información respecto de la plataforma English Discoveries Online, se puede encontrar en: http://queulatpre.usach.cl/blearning/sites/blearning/files/ manual2ode20usuario201.compressed.pdf

4. La responsabilidad del PIBL a nivel institucional corresponde a la Vicerrectoría Académica de la Universidad de Santiago de Chile, no obstante, su funcionamiento, es responsabilidad de los académicos que el Departamento de Lingüística y Literatura designa para su coordinación.

5. Las habilidades consideradas en esta pregunta fueron seleccionadas de los descriptores del nivel B1 del Marco Común Europeo en el proceso de validación por juicio. El criterio de elegibilidad se orientó al núcleo de habilidades fundamentales que estuvieran más relacionadas con los objetivos del programa y de sus componentes.

\section{REFERENCIAS}

Anderson, T. (2008). The theory and practice of online learning. Edmonton: AU Press, Athabasca University.

Bañados, E. (2006). A Blended-Learning Pedagogical Model for Teaching and Learning EFL Successfully through an Online Interactive Multimedia Environment. CALICO Journal, 23(3), 533-550. doi: https://doi.org/10.1558/ cj.v23i3.533-550

Benavides, M., y Gómez-Restrepo, C. (2005). Métodos en investigación cualitativa: triangulación. Revista Colombiana de Psiquiatría, 34(1), 118-124. Recuperado de: $\quad$ https://www.redalyc.org/articulo. oa? id $=80628403009$

Beetham, H., y Sharpe, R. (2007). Rethinking Pedagogy for a Digital Age: Designing and Delivering E-Learning. London: Routledge

Council of Europe (2018). Common European Framework of Reference for Languages: learning, teaching, assessment companion Volume with new descriptors. Recuperado de https:// rm.coe.int/cefr-companion-volume-withnew-descriptors-2018/1680787989
Didou, S., y Jaramillo, V. (2014). Internacionalización de la educación superior y las ciencias en América Latina: un estado del arte. Caracas: UNESCO.

Ferreira, A., Vine, A. y Elejalde, J. (2015). Diseño y evaluación de una aplicación tecnológica para la enseñanza del español como lengua extranjera. Onomázein, 31, 145-166. doi: https://doi.org/10.7764/ onomazein.31.10

Finardi, K., Vieira, G., y Schmitt, J. (2016). English distance learning: possibilities and limitations of MEO for the Flipped Classroom. RBLA, Revista Brasileira de Linguística Aplicada, 16(2), 181-208. doi: $\quad$ https://doi.org/10.1590/1984$\underline{639820158527}$

García, J. (2000). Entrenamiento en estrategias de aprendizaje de inglés como lengua extranjera en un contexto de aprendizaje combinado. Revista Nebrija de Lingüística Aplicada, 8(4), 17-40. Recuperado de https://www.nebrija. com/revista-linguistica/entrenamientoen-estrategias-de-aprendizaje-de-inglescomo-lengua-extranjera-en-un-contextode-aprendizaje-combinado.html 
García, J., Ferreira, A., y Morales, S. (2011). Autonomía en el aprendizaje de lenguas extranjeras en contextos de enseñanza mediatizados por la tecnología. Onomázein, 25(1), 15-50. Recuperado de https://www.redalyc.org/ pdf/1345/134524361001.pdf

Gass, S. (1997). Input, interaction, and the second language learner. Mahwah, NJ: Lawrence Erlbaum.

Gass, S., y Selinker, L. (2008). Second language acquisition: An introductory course. New York: Routledge.

Gómez, I., Hernández, E., y Rico, M. (2009). Moodle en la enseñanza presencial y mixta del inglés en contextos universitarios. RIED Revista Iberoamericana de Educación a Distancia, 12(1), 169193. doi: https://doi.org/10.5944/ ried.1.12.926

Graham, C., Woodfield, W., y Harrison, J. (2013). A framework for institutional adoption and implementation of blended learning in higher education. The Internet and Higher Education, 18, 4-14. doi: http://dx.doi.org/10.1016/j. iheduc.2012.09.003

Güzer, B., y Caner, H. (2013). The past, present and future of blended learning: an in depth analysis of literature. 5th World Conference on Educational Sciences - WCES 2013. Procedia-Social and Behavioural Sciences, 116, 45964603. doi: https://doi.org/10.1016/j. sbspro.2014.01.992

King, S., y Arnold, K. (2012). Blended learning environments in higher education: a case study of how professors make it happen. Mid-Western Educational Researcher, 25, 44-59. Recuperado de https:// www.mwera.org/MWER/volumes/ v25/issue1-2/v25n1-2-King-ArnoldGRADUATE-STUDENT-SECTION.pdf

Marsh, D., y Johnson, C. (2013). The Laureate English Program: Taking a research informed approach to blended learning. Higher Learning Research
Communications, 3(1), 45-55. doi: https://doi.org/10.18870/hlrc.v3i1.103

McGee, P., y Reis, A. (2012). Blended Course Design: A Synthesis of Best Practices. Journal of Asynchronous Learning Networks, 16(4), 7-22. doi: https://doi. org/10.24059/olj.v16i4.239

Means, B., Toyama, Y., Murphy, R., y Baki, M. (2013). The effectiveness of online and blended learning: a meta-analysis of the empirical literature. Teachers College Record, 115(3), 1-47. Recuperado de https://www.sri.com/sites/default/files/ publications/effectiveness of online and blended learning.pdf

Morales, S., y Ferreira, A. (2008). La efectividad de un Modelo de Aprendizaje Combinado para la enseñanza del inglés como lengua extranjera: Estudio Empírico. RLA. Revista de Lingüística Teórica y Aplicada, 46(2), 95-118. doi: $\quad$ https://doi.org/10.4067/So71848832008000200006

Leow, R. (2015). Explicit learning in the L2 classroom: a student-centered approach. New York: Routledge.

Norris, J. (2016). Language Program Evaluation. The Modern Language Journal, 10o(51), 169-189. doi: https:// doi.org/10.1111/modl.12307

Organización para la Cooperación y el Desarrollo Económicos (OCDE). (2009). La educación superior en Chile. Santiago: OCDE-BM.

Porter, W., Graham, C., Spring, K., y Welch, $\mathrm{K}$. (2014). Blended learning in higher education: Institutional adoption and implementation. Computers \& Education, 75, 185-195. doi: https://doi. org/10.1016/j.compedu.2014.02.011

Robinson, P. (1995). Attention, memory and the 'noticing' hypothesis. Language Learning, 45(2), 283-331. doi: https:// doi.org/10.1111/j.1467-1770.1995. tbo0441.x

Serag, A. (2017). Cultural Differences: Technology and the Design of EFL 
Learning Environment in the Japanese Context. En J. Colpaert, A. Aerts, R. Kern y M. Kaiser (Ed.), CALL in Context (666681). Berkeley: UCLA.

Sharpe, R., Benfield, G., Roberts, G., y Francis, R. (2006). The undergraduate experience of blended e-learning: A review of UK literature and practice. The Higher Education Academy. Recuperado de $\quad$ https://www.heacademy.ac.uk/ system/files/sharpe benfield roberts francis o.pdf

Strauss, A., y Corbin, J. (2002). Bases de la investigación cualitativa: técnicas y procedimientos para desarrollar la teoría fundamentada. Antioquía: Universidad de Antioquía.

Tomlinson, B., y Whittaker, C. (2013). Blended Learning in English Language Teaching: Course Design and Implementation. London: British Council.

VanPatten, B. (2004). Processing instruction: Theory, research, and commentary. Mahwah, NJ: Lawrence Erlbaum.

Warren, D. (2002). Curriculum design in a Context of Widening Participation in Higher Education. Arts and Humanities in HigherEducation, 1(1),85-99.doi:https:// doi.org/10.1177/1474022202001001007

\section{PERFIL ACADÉMICO Y PROFESIONAL DE LOS AUTORES}

Rodolfo Martinic Lenta es doctorando en Sociología, es investigador en trayectorias educativas y laborales y evalaudor de programas educativos en el École des hautes études en sciences sociales, en Francia. https://orcid.org/0000-00017179-319X?lang=en.

E-mail: rodolfo.martinic@gmail.com

Dirección:

23 rue Émile Lepeu, 75011. Paris, Francia

Sergio Urzúa Martínez tiene un Magíster en Sociología y Magíster en Políticas Públicas y es investigador en políticas de equidad y programas de inclusión en educación superior en la Universidad de Santiago, en Chile. https://orcid.org/oooo0002-6668-7170.

Email: sergio.urzua@usach.cl

Dirección:

Perpetua Freire 821.

La Florida. Santiago, Chile.

Ricardo Úbeda Menichetti tiene un Magíster en Lingüística Inglesa y es Profesor del Departamento de Lingüística y Literatura de la Universidad de Santiago en Chile.

E-mail: ricardo.ubeda@usach.cl 
Dirección:

Libertador Bernardo O’Higgins 3363. Estación Central. Santiago, Chile.

Roxana Aranda Gengler es Profesora de Física y Matemática y coordinadora del Área de evaluación VRA en la Universidad de Santiago, en Chile.

E-mail: roxana.aranda@usach.cl

Dirección:

Pica 1031. Las Condes.

Santiago, Chile.

Fecha de recepción del artículo: 05/12/2018

Fecha de aceptación del artículo: 04/03/2018

Fecha de aprobación para maquetación: 13/05/2019 